\title{
Overexpressed hPTTG1 promotes breast cancer cell invasion and metastasis by regulating GEF-H1/RhoA signalling
}

\author{
YC Liao', JW Ruan', I Lua ${ }^{2}, \mathrm{MH} \mathrm{Li}{ }^{3}$, WL Chen ${ }^{4}$, JRY Wang 5 , RH Kao ${ }^{5}$ and JH Chen ${ }^{1,2}$ \\ ${ }^{1}$ Institute of Medical Science, Tzu-Chi University, Hualien, Taiwan; ${ }^{2}$ Department of Molecular Biology and Human Genetics, Tzu-Chi \\ University, Hualien, Taiwan; ${ }^{3}$ Department of Pathology, Buddhist Tzu-Chi General Hospital, Hualien, Taiwan; ${ }^{4}$ Graduate Institute of \\ Life Science, National Defense Medical Center, Taipei, Taiwan and ${ }^{5}$ Department of Hematology-Oncology, Buddhist Tzu-Chi \\ General Hospital, Hualien, Taiwan
}

\begin{abstract}
Human pituitary tumour-transforming gene 1 (hPTTG1) is an oncogenic transcription factor that is overexpressed in many tumour types, especially tumours with metastatic abilities. However, how hPTTG1 overexpression drives metastasis is not yet clear. As a transcription factor, hPTTG1 may promote metastasis by activating target genes that are involved in the metastatic process. Here, we showed that Rho guanine nucleotide exchange factor-H1 (GEF-H1) was transcriptionally activated by hPTTG1, thereby promoting breast cancer metastasis. Luciferase reporter analyses and chromatin immunoprecipitation (ChIP) assays showed that hPTTG1 directly bound and activated the GEF-H1 gene promoter. In this study, RNA interference-mediated knockdown of hPTTG1 in highly metastatic breast tumour cells decreased GEF-H1 expression and RhoA activation, thereby reducing cell motility and invasion, and interfering with cytoskeletal remodelling in vitro, and impairing the tumour metastasis in vivo. The restoration of GEF-H1 expression in hPTTG1-knockdown cells rescued the hPTTG1-knockdown effects on cytoskeletal changes in vitro and tumour metastasis in vivo. Conversely, ectopic expression of hPTTG1 in non-metastatic breast tumour cells induced cytoskeletal rearrangements, and allowed these cells to metastasise in a mouse model by orthotopic implantation. In human tumour samples, hPTTG1 expression was also correlated to GEF-H1 expression in aggressive breast carcinoma. Altogether, these findings definitively establish a role for hPTTG1 in activating the GEF-H1/RhoA pathway as a newly identified mechanism in breast cancer metastasis. Oncogene (2012) 31, 3086-3097; doi:10.1038/onc.2011.476; published online 17 October 2011
\end{abstract}

Keywords: hPTTG1; GEF-H1; RhoA; tumour metastasis; cytoskeleton regulation

Correspondence: Professor JH Chen, Department of Molecular Biology and Human Genetics, Tzu Chi University, Rm. D416, No.701, Sec. 3, Zhongyang Rd., Hualien, Hualien County 97004, Taiwan.

E-mail: noli401029@gmail.com or lecoisoft@gmail.com

Received 20 January 2011; revised 21 June 2011; accepted 9 September 2011; published online 17 October 2011

\section{Introduction}

Metastasis is the major cause of death in cancer patients (Gupta and Massague, 2006), but how these tumour cells become metastatic is not wholly understood. The invasion-metastasis cascade is a multistep process, involving the invasion of tumour cells into adjacent sites, intravasation into the systemic circulation, survival of tumour cells during the stress of circulation, extravasation into distant tissues, and establishment of micrometastases and outgrowth of secondary tumours (Chambers et al., 2002; Fidler, 2003).

Human pituitary tumour-transforming gene 1 ( $h P T T G 1$ ), which encodes the human securin protein (Pei and Melmed, 1997; Zou et al., 1999) is a multifunctional gene. In cell-cycle regulation, hPTTG1 controls sister chromatid separation during mitosis (Funabiki et al., 1996; Hornig et al., 2002). Moreover, hPTTG1 protein can interact with a variety of proteins that are important for the regulation of cellular circuitry; these proteins include PBF, PP2A, Ku70 and p53 (Chien and Pei, 2000; Romero et al., 2001; Bernal et al., 2002; Gil-Bernabe et al., 2006). For example, hPTTG1 regulates DNA-damage repair mechanisms through its interaction with $\mathrm{Ku} 70$ protein and inhibits the $\mathrm{Ku} 70$-dependent repair process (Romero et al., 2001). hPTTG1 has also been characterised as a transcription factor that regulates the expression of genes involved in a variety of cellular processes (Wang and Melmed, 2000; Vlotides et al., 2007; Tong and Eigler, 2009). hPTTG1 transcriptionally activates oncogenes such as c-myc and fibroblast growth factor (FGF)2 (Chien and Pei, 2000; Pei, 2001), but detailed mechanisms of how hPTTG1 promotes metastasis through the induction of these target genes are still unclear.

hPTTG1 behaves as an oncogene whose overexpression transforms fibroblasts in vitro and enhances tumourigenesis in vivo (Zhang et al., 1999; Zou et al., 1999). In several clinical studies, hPTTG1 was found to be overexpressed in most invasive solid tumours and blastic leukaemias (Dominguez et al., 1998; Heaney et al., 2000; Ogbagabriel et al., 2005; Ito et al., 2008). It is also recognised as one of the 'signature molecules' for metastasis (Ramaswamy et al., 2003). hPTTG1 overexpression is considered a potential marker that is 
relevant to the malignancy of breast cancer (Solbach et al., 2004; Ogbagabriel et al., 2005). However, how overexpressed hPTTG1 promotes breast cancer metastasis remains unknown.

We hypothesised that hPTTG1 exerts its metastasispromoting functions by acting as a transcriptional activator for genes involved in the metastatic process. Here, we found that hPTTG1 directly regulated the transcription of guanine nucleotide exchange factor-H1 (GEF-H1), which is an oncoprotein that specifically activates RhoA (Ren et al., 1998), a well-known member of the Rho family of GTPases. Activated RhoA mediates multiple signals that are involved in cytoskeleton organisation, cell motility, cell invasion and cell proliferation (Hall, 1998; Schmitz et al., 2000; Ridley, 2001; Mizuarai et al., 2006). All of these events contribute to tumour metastasis (del Peso et al., 1997; Jaffe and Hall, 2005). Although the role of RhoA in cancer development and metastasis has been thoroughly investigated in recent years (Valastyan et al., 2009; Chan et al., 2010), the upstream regulation of GEF-mediated RhoA activation is still unclear. In this study, we show that hPTTG1 is a transcription factor that triggers the GEF-H1/RhoA pathway to accelerate breast cancer invasion and metastasis. In human invasive breast carcinoma, we also found that hPTTG1 is overexpressed and is correlated to GEF-H1 expression.

\section{Results}

\section{hPTTG1 level affects GEF-H1 expression in breast} cancer cell lines

To search for putative genes regulated by hPTTG1, we compared the expression profile of $h P T T G 1$-null HCT116 cells with those cells overexpressing $h P T T G 1$ (Supplementary Figure S1a). There were 49 genes that were upregulated by at least threefold in $h P T T G 1$-null HCT116 and $h P T T G 1$-overexpressing cells when compared with $h P T T G 1$-null HCT116 cells. Among these upregulated genes, seven genes are involved in tumourigenesis (Supplementary Table S1). To investigate the role of hPTTG1 in tumour metastasis, we focused on GEF-H1, whose activity is regulated by microtubule dynamics in cytoskeleton organisation (Krendel et al., 2002) and is associated with tumourigenesis (Mizuarai et al., 2006). Hence, we validated the messenger RNA (mRNA) and protein expression levels of GEF-H1 in hPTTG1-overexpressed cells by real-time PCR and immunoblot analysis (Supplementary Figures S1a and $b$ ).

Increased expression of hPTTG1 is a risk factor of breast cancer (Ogbagabriel et al., 2005). To investigate the association of hPTTG1 and GEF-H1 in breast cancer, we examined the expression status of hPTTG1 and GEF-H1 in eight breast cancer cell lines: normal human mammary epithelial cells (H184B5F5/M10), five non-metastatic cancer cell lines (BT-483, Au565, T-47D, ZR75-1 and MCF-7) and two metastatic cancer cell lines (MDA-MB-231 and MDA-MB-435). Higher levels of hPTTG1 protein were observed in most breast cancer cell lines when compared with H184B5F5/M10 normal mammary epithelial cells (Figure 1a; Supplementary Figure S2a). Quantification of western blots indicated that hPTTG1 protein was expressed more highly (ranging from 1.3-fold to 3.4-fold) in breast cancer cell lines than normal mammary epithelial cells (Supplementary Figure S2a). Similarly, most breast cancer cell lines expressed higher levels of both GEF-H1 mRNA (ranged from 2- to 4-fold) and protein (ranging from 7to 20 -fold), especially in metastatic cancer cell lines, when compared with normal epithelial mammary cells (Figure 1a; Supplementary Figure S2b). These results indicate that the expression level of hPTTG1 affects the expression of GEF-H1 in breast cancer cell lines, especially in metastatic breast cancer cells.

\section{hPTTG1 functions as a transcription factor to transactivate the GEF-H1 gene in breast cancer cells} To investigate whether hPTTG1 regulates GEF-H1 transcription, we cloned the regulatory sequence of the GEF-HI gene (from $1600 \mathrm{bp}$ upstream to $98 \mathrm{bp}$ downstream of the transcription start site) into the pGL3 luciferase vector for luciferase reporter assays. We found that GEF-H1 transcriptional activity was enhanced by hPTTG1 overexpression (Figure 1b). This result suggests that hPTTG1 regulates GEF-H1 by transcriptional activation.

hPTTG1 is a functional transcription factor, and the sequence of a hPTTG1-binding element has been identified as TTATCT (Pei, 2001). Based on this sequence, we found that the promoter region of GEF-H1 contained three putative (TTaACT, TTcTCT and TTATgT) hPTTG1-binding elements (Figure 1b). To confirm whether hPTTG1 activated GEF-H1 transcription through any of these three putative binding elements directly, we generated two truncation mutants with $5^{\prime}$ deletions of the GEF-H1 promoter (Figure 1c). Our results show that the transcriptional activity of fragment $\mathrm{B}$, which contains putative hPTTG1-binding elements 2 and 3, was not induced by hPTTG1 (Figure 1c), whereas the transcriptional activity of fragment $\mathrm{C}$, which contains only putative hPTTG1-binding element 3 , was induced by hPTTG1 slightly, suggesting that hPTTG1 may regulate GEF-H1 transcription by binding on putative binding element 1 or 3 . Next, we used electrophoretic mobility shift assay (EMSA) to assess whether hPTTG1 binds to the GEF-H1 promoter. Addition of the $-1160 /-1140$ probe to recombinant hPTTG1 protein formed specific binding complex that was competed by homologous competitors (Supplementary Figure S3, lanes 2-3). However, addition of the $-1005 /-986$ and $-275 /-257$ probes to recombinant hPTTG1 protein did not form binding complex (Supplementary Figure S3, lanes 6-7). These results suggest that the promoter region between -1160 and $-1140 \mathrm{bp}$ may be critical for hPTTG1 binding, and this region contains putative hPTTG1-binding element 1 .

To verify the possibility that putative hPTTG1binding element 1 is critical for GEF-H1 transcription, we mutated putative hPTTG1-binding element 1 and analysed its reporter activities. Mutation of putative hPTTG1-binding element 1 significantly attenuated the hPTTG1-induced activation of the GEF-H1 promoter 
a

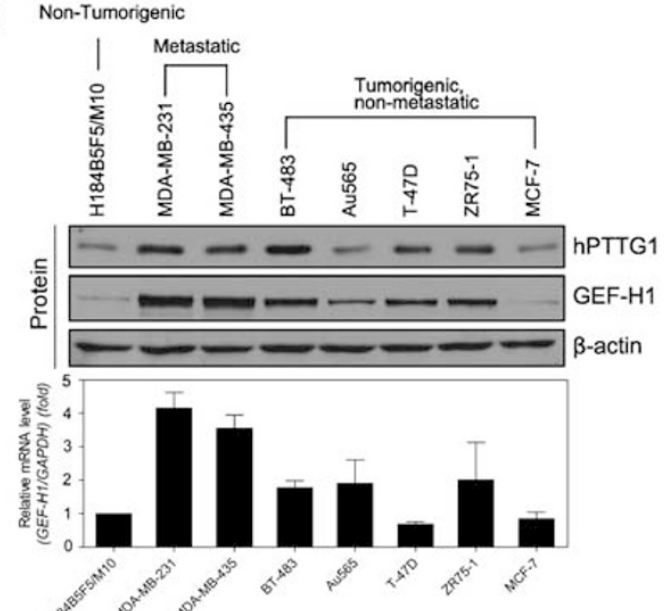

b

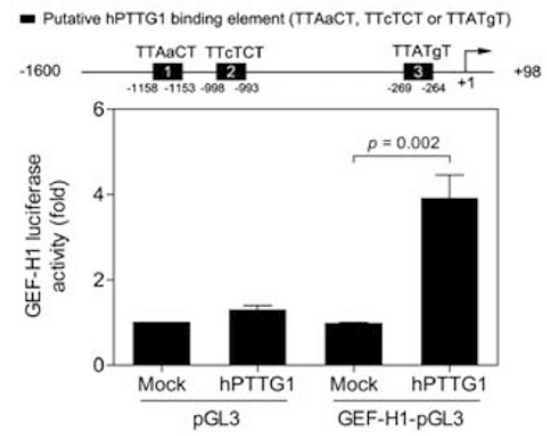

C
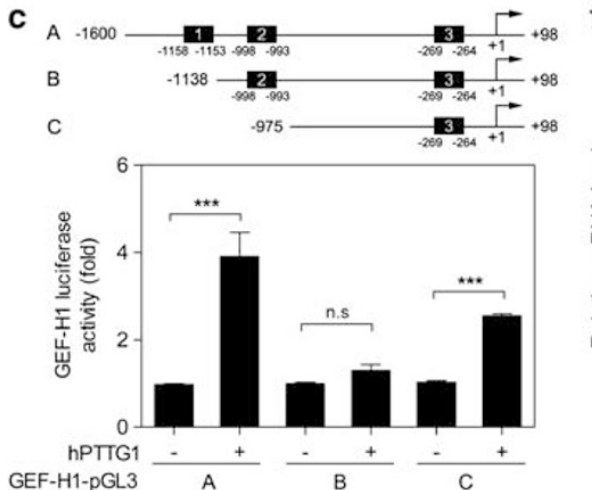

d
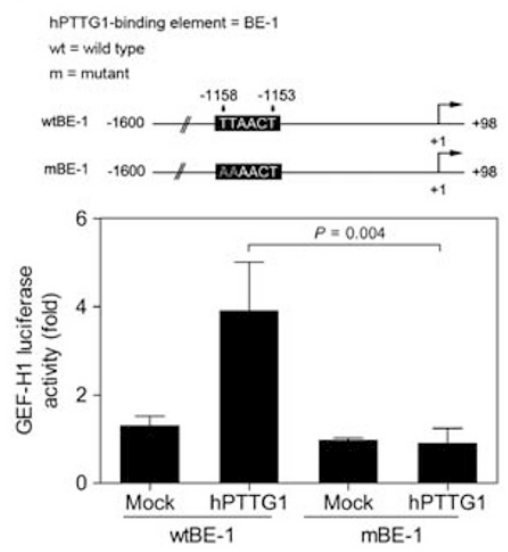

e

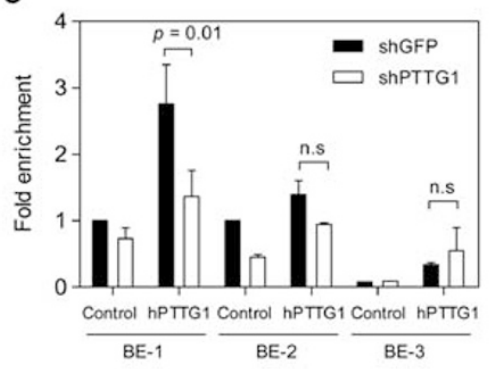

f $\quad$ g

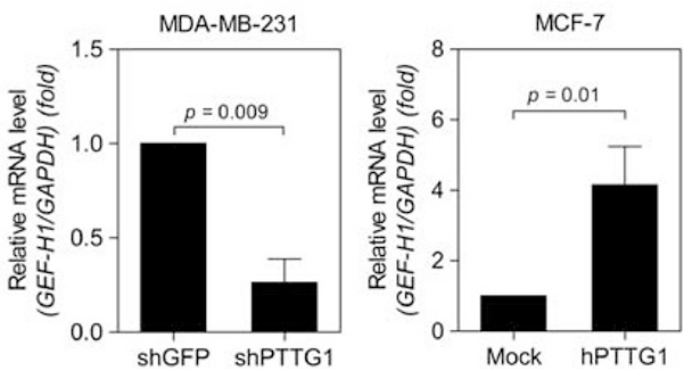

Figure 1 hPTTG1 is a transcriptional activator of GEF-H1. (a) Immunoblot analysis of hPTTG1 and GEF-H1 expression in all eight cell lines. $\beta$-Actin was used as a loading control (top). The transcript level of GEF-H1 was analysed by real-time PCR. GAPDH was used as the loading control (bottom). (b) The GEF-H1 regulatory sequence contains three putative hPTTG1-binding elements (binding element 1: nucleotides -1158 to -1153 ; binding element 2: nucleotides -998 to -993 ; binding element 3: nucleotides -269 to -264 ) (top). hPTTG1 activates the GEF-H1 promoter in MCF-7 cells. The GEF-H1 promoter was co-transfected with pcDNA3.1 vector (Mock) or pcDNA3.1-hPTTG1 expression plasmid, and harvested for luciferase reporter assays (bottom). (c) A series of GEF-H1 promoter deletion mutants (fragments B and C, top) were used for luciferase reporter assays in MCF-7 cells (bottom). (d) A sitespecific mutation (TTAACT AAAACT) was introduced in the hPTTG1-binding element 1 on the GEF-H1 promoter and was cotransfected with pcDNA3.1 vector (Mock) or pcDNA3.1-hPTTG1 expression plasmid in MCF-7 cells, and luciferase reporter assays were performed. (e) ChIP assays were performed in MDA-MB-231 cells with GFP or hPTTG1 knockdown (hPTTG1 knockdown efficiency is shown in Figure 2a). The ChIP-qPCR data are expressed as the fold increase over the control (IgG) on the promoter region containing hPTTG1-binding elements 1, 2 and 3. (f) GEF-H1 mRNA expression was analysed by real-time PCR in MDA-MB-231 cells with GFP or PTTG1 knockdown, and in (g) MCF-7 cells transfected with pcDNA3.1 vector (Mock) or pcDNA3.1-hPTTG1. Human GAPDH was used as the loading control. Results are presented as mean \pm s.e.m. $(n=3)$. $* * *<0.0001$; n.s., not significant.

(Figure 1d), suggesting that hPTTG1 regulates GEF-H1 transcription through binding element 1 . Further, a chromatin immunoprecipitation (ChIP) assay was performed in vivo to determine whether hPTTG1 interacts with the endogenous GEF-H1 promoter in MDA-MB-
231 cells. The ChIP assay revealed that the promoter region containing the element 1 was specifically coimmunoprecipitated by anti-hPTTG1 antibody, whereas hPTTG1 knockdown significantly attenuated this effect (Figure 1e). We found that hPTTG1 also had slight 
binding capacity to element 2 , but the effect was not significantly affected by hPTTG1 knockdown (Figure 1e). However, hPTTG1 had no binding capacity to element 3. Although the above luciferase assay showed that element 3 may function to regulate GEFH1 transcription, this result may have occurred because fragment $\mathrm{C}$ contained other transcription factor sites. These results suggest that hPTTG1 could directly bind to the GEF-H1 promoter at element 1. Furthermore, real-time PCR revealed that GEF-H1 mRNA expression was decreased in hPTTG1-knockdown MDA-MB231 cells (Figure 1f); conversely, GEF-H1 mRNA expression was significantly induced by hPTTG1 overexpression in MCF-7 cells (Figure 1g). Altogether, these results suggested that hPTTG1 functions as a transcription factor to directly activate GEF-H1 transcription.

\section{GEF-H1 mediates hPTTG1-enhanced breast cancer cell migration and invasion}

We next examined whether the hPTTG1 level is critical for GEF-H1 protein expression. Metastatic MDA-MB231 and non-metastatic MCF-7 cells were utilised as in vitro experimental models. MDA-MB-231 cells had significant metastatic potential in vitro and in vivo, and expressed high levels of endogenous hPTTG1 and GEFH1 proteins (Figure 1a). Conversely, MCF-7 cells expressed low levels of endogenous hPTTG1 and GEF-H1 (Figure 1a). Knockdown of hPTTG1 expression by specific shRNAs in MDA-MB-231 cells resulted in decreased GEF-H1 protein expression (Figure 2a). In contrast, ectopic hPTTG1 expression in MCF-7 cells profoundly increased GEF-H1 expression (Figure 2b). As GEF-H1 is a specific guanine exchange factor for RhoA-GTPase (Ren et al., 1998), we further confirmed whether the hPTTG1/GEF-H1 axis affects RhoA activity. Indeed, the amount of activated RhoA-GTP was reduced in hPTTG1-knockdown MDA-MB-231 cells, and was greatly increased by hPTTG1 overexpression in MCF-7 cells (Figures 2c and d).

The specific role of RhoA in the acquisition of mobile properties and invasive capacities in tumour cells has been well-documented (Schmitz et al., 2000; Ridley, 2001). Thus, we hypothesised that hPTTG1/GEF-H1 signalling may be critical for cancer cell migration, invasion and metastasis by activating RhoA. Indeed, hPTTG1 knockdown reduced cell migration and invasion in MDA-MB-231 cells (Figure 3a), whereas hPTTG1 overexpression promoted cell migration and invasion in MCF-7 cells (Figure 3b). hPTTG1 protein transfection also rescued the ability of hPTTG1-knockdown MDA-MB-231 cells to migrate and invade (Supplementary Figure S4a). GEF-H1 knockdown or overexpression phenocopied the effects of hPTTG1 on migration and invasion (Figure 3c; Supplementary Figure S4b), indicating that hPTTG1 may regulate these processes by upregulating GEF-H1. To test this idea, we restored GEF-H1 expression in hPTTG1knockdown MDA-MB-231 cells and found that restoration of GEF-H1 expression rescued the defects of cell migration and invasion caused by hPTTG1 knockdown (Figure 3d). The effects of hPTTG1 and GEF-H1 knockdown on cell migration and invasion were due to the biological activities of hPTTG1 and GEF-H1, as hPTTG1 and GEF-H1 knockdown did not affect cell proliferation or cell cycle progression (Supplementary Figures $\mathrm{S} 5 \mathrm{a}-\mathrm{c})$. Our results therefore suggest that hPTTG1 enhances breast cancer cell migration and invasion by inducing GEF-H1 signalling.

\section{hPTTG1 regulates actin cytoskeleton rearrangements by GEF-H1 signalling}

As GEF-H1 activity is critical for RhoA activation, GEF-H1 may regulate several RhoA-dependent biological processes associated with the actin cytoskeleton, such as cell morphology, polarity and motility (Hall, 1998; Sahai and Marshall, 2002). Modulation of RhoA activity regulates cell retraction through the assembly of F-actin stress fibres and focal adhesions, two key cytoskeletal events of migrating cells (Wittmann and Waterman-Storer, 2001). To investigate whether hPTTG1 regulates changes in the actin cytoskeleton to enhance breast cancer cell motility, we monitored the alterations of stress fibres and focal adhesions by staining for F-actin and paxillin, respectively. Paxillin is a focal adhesion-associated protein and is required for regulating cell spreading and motility (Schaller,
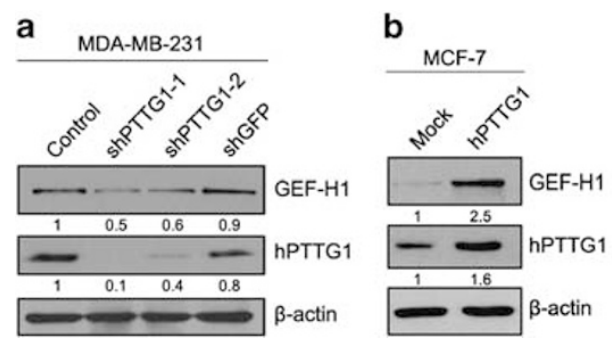

C

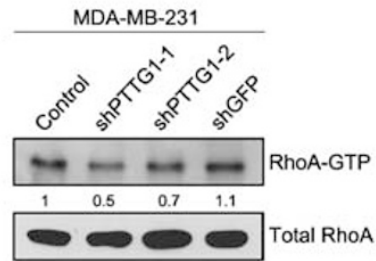

d

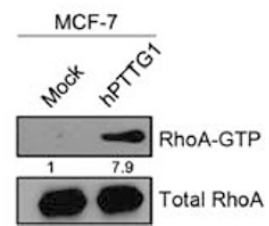

Figure 2 hPTTG1 regulates the expression of GEF-H1 and the activation of RhoA. (a) Immunoblot analysis of hPTTG1 and GEFH1 expression in control (not transfected with any DNA), GFP-knockdown or hPTTG1-knockdown MDA-MB-231 cells. (b) Immunoblot analysis of hPTTG1 and GEF-H1 expression in MCF-7 cells transfected with pcDNA3.1 vector (Mock) or pcDNA3.1-hPTTG1 expression plasmid. (c) Immunoblot analysis of active RhoA (RhoA-GTP) and total in control (not transfected with any DNA), GFP-knockdown or hPTTG1-knockdown MDA-MB-231 cells. (d) Immnuoblot analysis of active RhoA (RhoAGTP) and total RhoA in MCF-7 cells transfected with pcDNA3.1 vector (Mock) or pcDNA3.1-hPTTG1 expression plasmid. The relative intensity of protein was quantified by Quantity One software and normalised to $\beta$ - actin and total RhoA. 

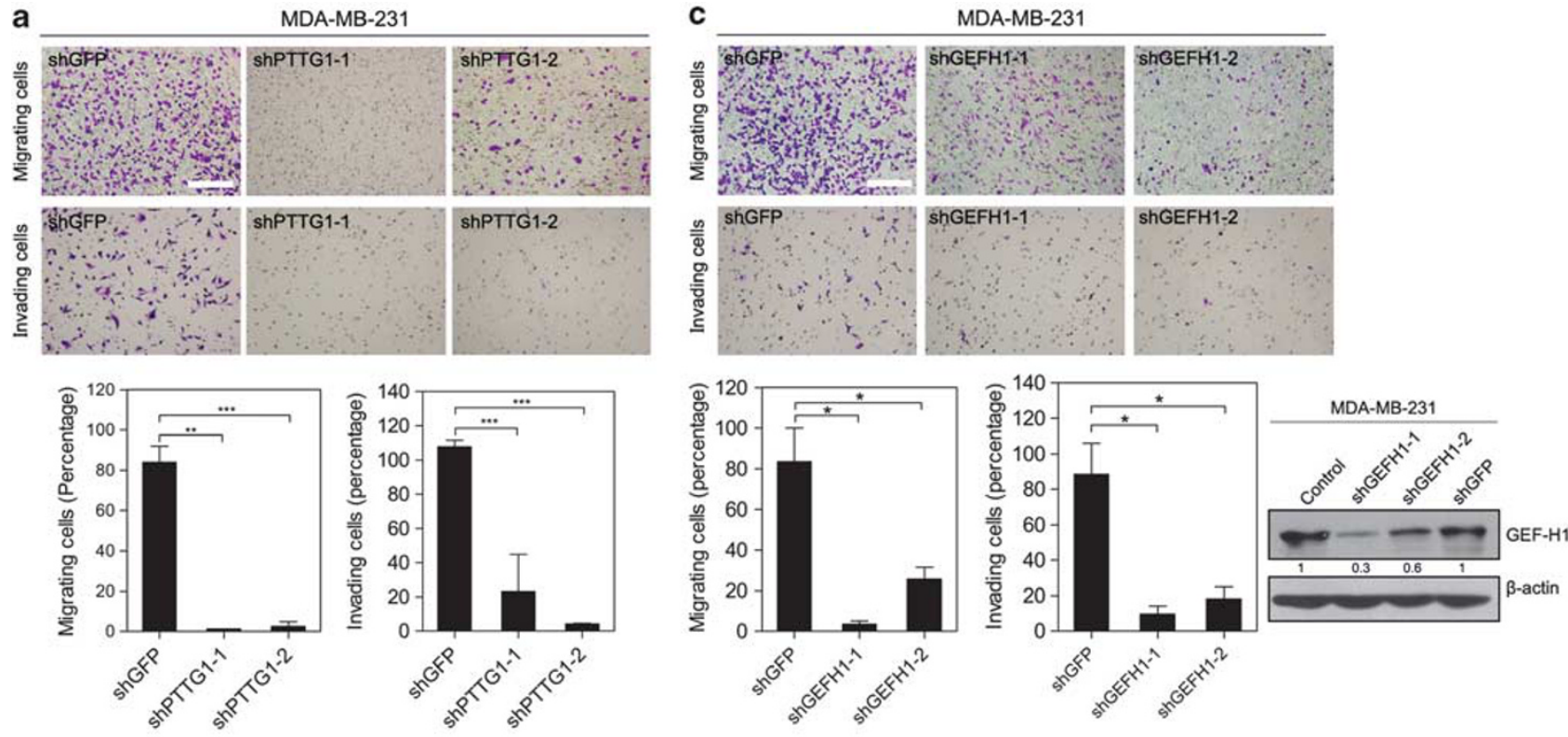

b
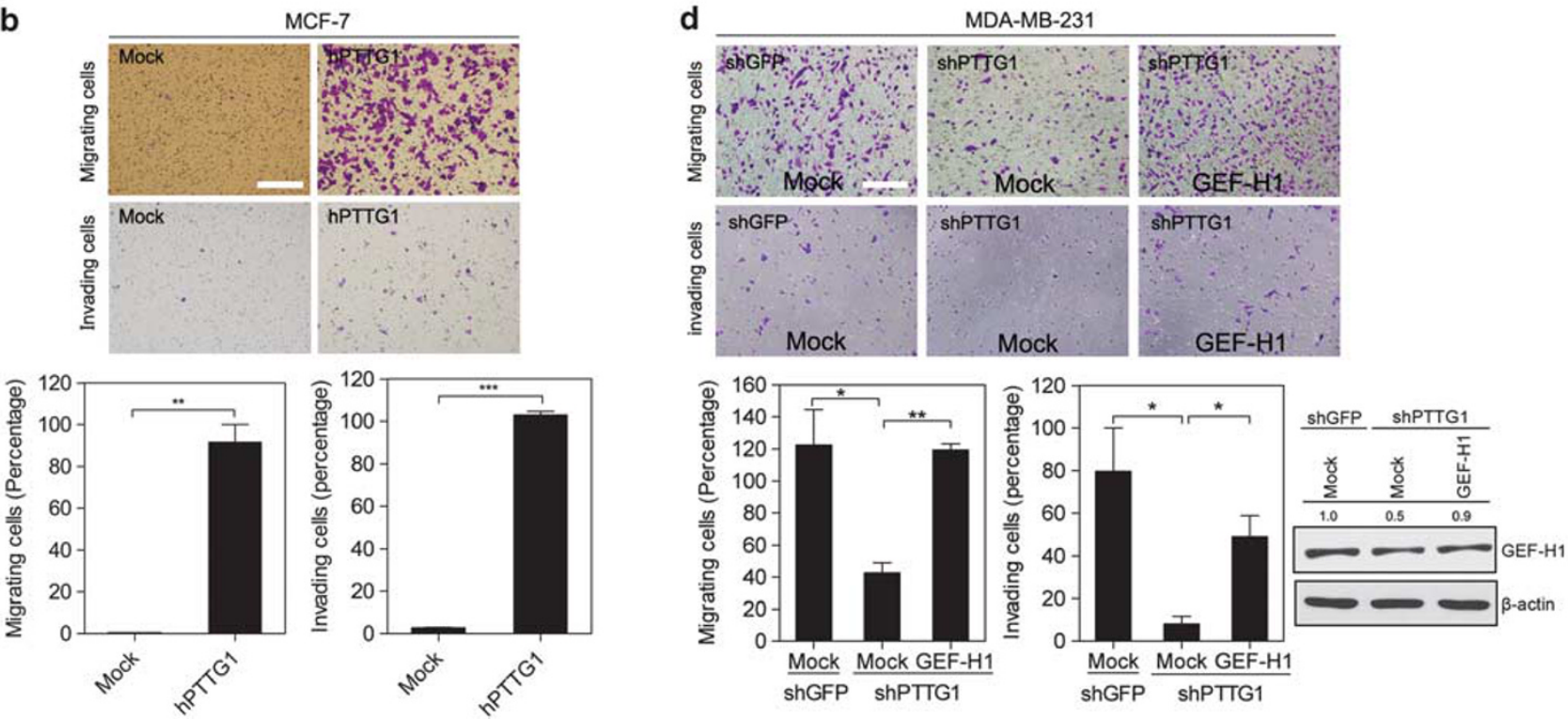

Figure 3 hPTTG1 regulates cell migration and invasion through GEF-H1. (a) Migration and invasion assays in MDA-MB-231 cells with GFP or hPTTG1 knockdown (hPTTG1 knockdown efficiency is shown in Figure 2a). (b) Migration and invasion assays in MCF-7 cells transfected with pcDNA3.1 vector (Mock) or pcDNA3.1-hPTTG1 expression plasmid (hPTTG1 overexpression efficiency is shown in Figure 2b). (c) Migration and invasion assays and immunoblot analysis in MDA-MB-231 cells with GFP or GEF-H1 knockdown. (d) Migration and invasion assays and immunoblot analysis in MDA-MB-231 cells with GFP or hPTTG1 knockdown or with hPTTG1 knockdown plus GEF-H1 overexpression. The relative intensity of protein was quantified by Quantity One software and normalised to $\beta$ - actin. Results are presented as mean \pm s.e.m. $(n=3) .{ }^{*} P<0.05 ; * * P<0.01 ; * * *<0.0001$. Images shown in Figure $3 \mathrm{a}-\mathrm{d}$ show the migrating and invading cells in polycarbonate membrane filters. Magnification, $\times 200$. Scale bars, $200 \mu \mathrm{m}$.

2001). Control shGFP-knockdown MDA-MB-231 cells displayed a mesenchymal and spreading morphology (Supplementary Figure S6; Figure 4A), with abundant stress fibres (Figure $4 \mathrm{Ab}$ ) and punctate peripheral paxillin staining (Figure 4Aa). However, hPTTG1knockdown MDA-MB-231 cells displayed epitheliallike morphology, and spreading behaviour was attenuated (Supplementary Figure S6; Figure 4A), with a reduction in punctate paxillin staining (Figure 4Ad) and stress fibres (Figure 4Ae). In MCF-7 cells, both
hPTTG1 and GEF-H1 overexpression induced a spreading morphology (Figure 4B), with an increase in punctate paxillin staining (Figure 4Bd and g) and stress fibres (Figure 4Be and h) when compared with control Mock-transfected MCF-7 cells (Figure 4Ba and b). Furthermore, we found that restoration of GEF-H1 expression rescued the cell-spreading defect in hPTTG1kockdown MDA-MB-231 cells, with increased punctate paxillin staining (Figure $4 \mathrm{Ag}$ ) and stress fibres (Figure 4Ah). These results suggest that the hPTTG1/ 
A
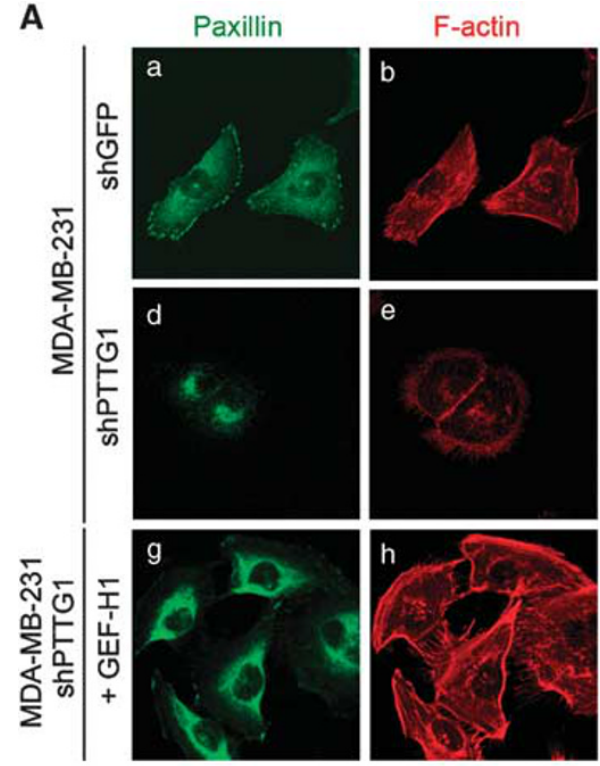

B
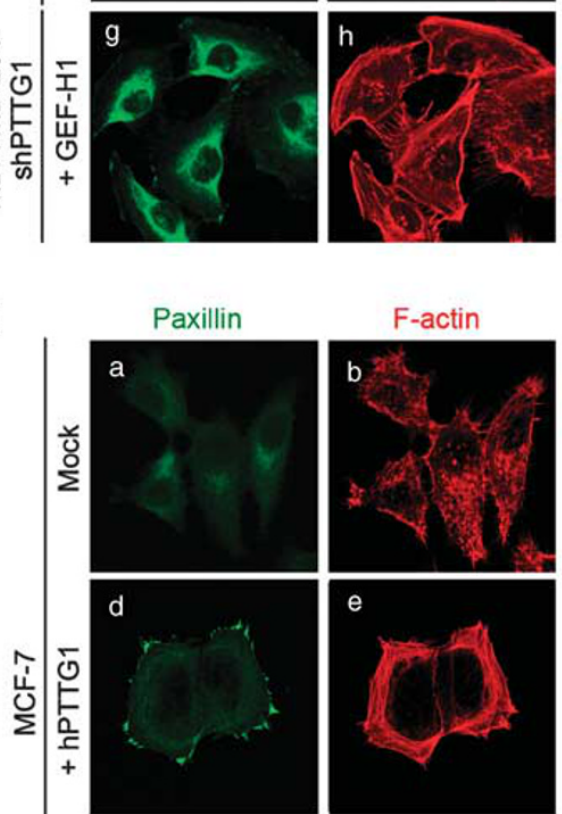

F-actin
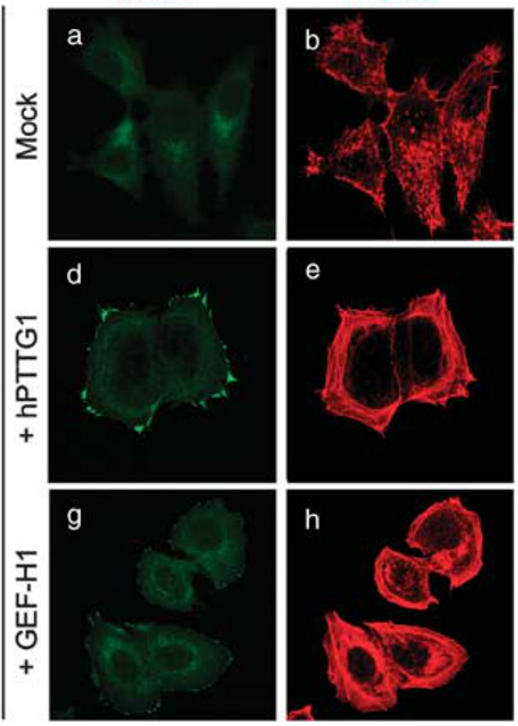

c
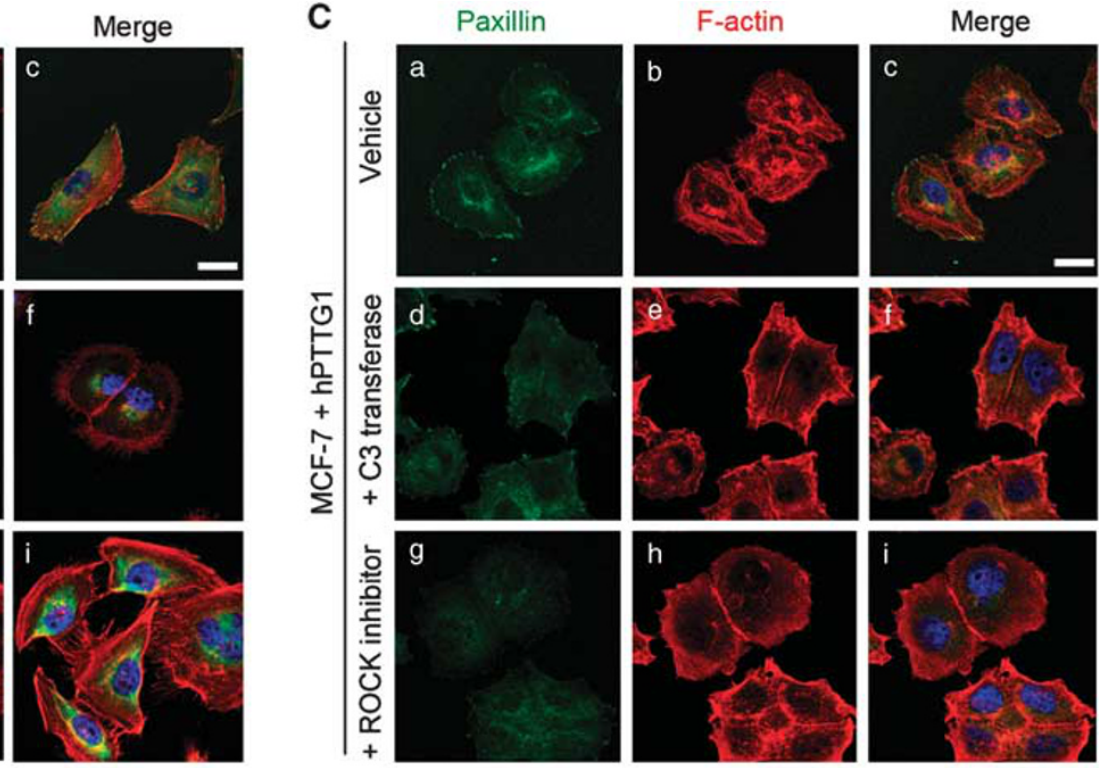
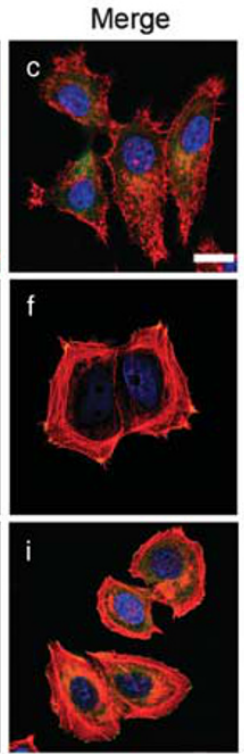

Figure 4 hPTTG1/GEF-H1 signalling regulates actin cytoskeleton rearrangements in breast cancer cells. (A) MDA-MB-231 cells with GFP or hPTTG1 knockdown or with hPTTG1 knockdown plus GEF-H1 overexpression were immunostained with anti-paxillin antibody (green) and phalloidin antibody (red) to observe focal adhesions and F-actin stress fibres, respectively. Depletion of hPTTG1 expression in MDA-MB-231 reduced the formation of focal adhesions (panel d), and F-actin stress fibres (panel e) when compared with control shGFPknockdown MDA-MB-231 cells (panels a, b). Ectopic expression of GEF-H1 in hPTTG1-knockdown MDA-MB-231 cells increased punctate paxillin staining (panel g) and F-actin stress fibres (panel h). (B) Ectopic expression of hPTTG1 and GEF-H1 in MCF-7 cells induces the formation of focal adhesions (panels d, g), and F-actin stress fibres (panels e, h) when compared with control MCF-7 cells transfected with pcDNA3.1 vector (Mock; panels a, b). (C) hPTTG1-overexpressed MCF-7 cells were plated on poly-L-lysine-coated coverslips for $48 \mathrm{~h}$ and then treated with C3 transferase (RhoA inhibitor, $2 \mu \mathrm{g} / \mathrm{ml}$ ) or 555550 (ROCK inhibitor, $10 \mu \mathrm{M}$ ) for 4 or $1.5 \mathrm{~h}$, respectively. The inhibitor-treated cells were then fixed and stained for DNA, focal adhesions (panels d, g) and F-actin (panels e, h). Both inhibitors impaired the phenotypes induced by hPTTG1. The vehicle-treated cells were shown in panels a-c. Scale bars, $20 \mu \mathrm{m}$. Merged images of paxillin and F-actin were shown in panels c, f and i of A-C. DAPI (blue) was used to visualize the nuclei.

GEF-H1 axis is important for regulating actin dynamics in breast cancer cells.

To support our in vitro findings that hPTTG1 overexpression could enhance RhoA activation, we used inhibitors of RhoA and its downstream molecule ROCK (C3 transferase and 555550, respectively) to treat hPTTG1-overexpressed MCF-7 cells and observe the alteration of actin cytoskeleton dynamics. The results show that the inhibition of RhoA signalling abolished hPTTG1-induced cytoskeletal phenotypes in hPTTG1-overexpressed MCF-7 cells (Figure 4C). (ROCK is a downstream RhoA effector that facilitates actin polymerisation and motility.) Both inhibitortreated cells displayed a reduction in punctate paxillin staining (Figure 4Cd and $\mathrm{g}$ ) and stress fibres (Figure 4Ce and $\mathrm{h}$ ), when compared with hPTTG1-overexpressed 
MCF-7 cells treated with vehicle (Figure $4 \mathrm{Ca}$ and $\mathrm{b}$ ). Our results indicate that the hPTTG1/GEF-H1 axis regulates actin dynamics by activating RhoA signalling, thus enhancing the motility and invasive potential of breast cancer cells.

\section{The hPTTG1/GEF-H1 axis is critical for breast cancer} metastasis in a mouse model

To further evaluate the role of the hPTTG1/GEF-H1 axis in mestastasis, we orthotopically injected the control shGFP-knockdown and hPTTG1-knockdown MDAMB-231 cells into the fourth mammary fat pads of severe combined immunodeficiency (SCID) mice and monitored tumour growths weekly. The results show that hPTTG1 knockdown did not affect primary tumour growth within the first 6 weeks, but it reduced the growth rate of tumours after the sixth week (Figure 5a). This finding supports the tumour-promoting properties of hPTTG1 in previous studies (Zou et al., 1999). Orthotopic injection of tumour cells can be used as a spontaneous metastatic mouse model to determine whether hPTTG1 influences the early steps of the invasion-metastasis cascade. hPTTG1-knockdown MDA-MB-231 cells were strikingly impaired in their capacity to seed lung metastases compared with control shGFP-knockdown MDA-MB231 cells (Figure 5b). hPTTG1-knockdown MDA-MB231 cells formed $80 \%$ fewer metastatic nodules than did controls 10 weeks after implantation.

Knockdown of hPTTG1 consistently decreased GEF$\mathrm{H} 1$ protein expression in both primary breast tumours (Supplementary Figures S7aD and E) and metastatic breast tumours within the lung (Supplementary Figures S7bC and D). We next asked whether ectopic hPTTG1 is required to induce metastasis. Thus, non-metastatic MCF-7 cells overexpressing hPTTG1 were also orthotopically injected into mice. hPTTG1-overexpressed MCF-7 cells metastasised to the lungs in significant numbers, whereas control Mock-transfected MCF-7 cells did not form metastatic nodules (Figure 5c). hPTTG1overexpressed MCF-7 cells formed 13-fold more metastatic nodules than did controls after 16 weeks after implantation. Overexpression of hPTTG1 also consistently increased GEF-H1 protein expression in both primary breast tumours (Supplementary Figures S7cD and E) and metastatic breast tumours within the lung (Supplementary Figures S6dC and D). However, RhoA expression was not induced either in MDA-MB-231 or in MCF-7 tumours (Supplementary Figures S7aC and $\mathrm{F}$, and $\mathrm{S} 7 \mathrm{cC}$ and $\mathrm{F}$ ). Our results suggest that hPTTG1 specifically upregulates GEF-H1 expression, thus promoting breast cancer cell invasion and metastasis in vivo.

To determine whether the impact of the hPTTG1/ GEF-H1 axis on metastasis also affects the later steps of the invasion-metastasis cascade, we injected the control and hPTTG1-knockdown MDA-MB-231 cells directly into mouse-tail veins, thereby preventing the early steps of local invasion and intravasation. After 10 weeks, mice injected with hPTTG1-knockdown MDA-MB-231 cells formed $86 \%$ fewer metastatic nodules than did control shGFP-knockdown MDA-MB-231 cells (Figure 5d).
Further, we found that restoration of GEF-H1 expression led to a recovery of $62 \%$ of the metastatic potential in hPTTG1-knockdown MDA-MB-231 cells (Figure 5d). These results suggest that the hPTTG1/GEF-H1 axis regulates the colonisation process of breast cancer cells into lung and promotes breast cancer metastasis in mice.

\section{hPTTG1 expression is correlated to GEF-H1 expression} in human invasive breast carcinoma

All of the results described above indicated that hPTTG1overexpression induced GEF-H1 expression in vitro, and that this signalling axis is critical for breast cancer invasion and metastasis in mice. Therefore, we investigated the clinical association of hPTTG1 and GEF-H1 in human breast cancer samples. As hPTTG1 transcriptionally activates GEF-H1, the prognostic significance of hPTTG1 was determined by assessing its nuclear staining (Ogbagabriel et al., 2005). Cancer samples were scored on the basis of the intensity of hPTTG1 nuclear staining and the percentage of hPTTG1-positive tumour cells; moreover, the intensity of GEF-H1 nuclear and cytoplasmic staining in tumour cells were analyzed (described in Materials and methods). Analyzing human invasive breast carcinomas (70 cases), hPTTG1 was predominantly expressed in the nuclei $(2+$ and $3+, 68$ out of 70$)$ (Figure 6Aa and b), whereas GEF-H1 had both a nuclear and cytoplasmic presence $(2+$ and $3+, 65$ out of 70$)$ (Figure 6Ad and e). However, in normal parts of these specimens, both hPTTG1 and GEF-H1 were expressed weakly in the cytoplasm (Supplementary Figure S8a). Indeed, in these 70 specimens, 64 samples showed abundant expression of both hPTTG1 and GEF-H1, and their expression levels were significantly associated with each other $(P=0.019)$ (Figure 6B). Among the hPTTG1-strong $(3+)$ group, $32 \%$ (8 out of 25$)$ of the samples showed GEF-H1-strong $(3+)$ expression, and $64 \%$ (16 out of 25) of the samples showed GEF-H1moderate $(2+)$ expression (Supplementary Figure S8b). In the hPTTG1-moderate $(2+)$ group, 79\% (34 out of $43)$ of the samples showed GEF-H1-moderate $(2+)$ expression (Supplementary Figure S8b).

Taken together, our results establish a molecular link between hPTTG1 and GEF-H1 in vitro and in vivo. The results of animal experiments and clinical investigations emphasise the importance of the hPTTG1/GEF-H1/ RhoA signalling axis in breast cancer metastasis.

\section{Discussion}

Our study identifies the role of overexpressed hPTTG1 in regulating breast cancer metastasis. First, we show that hPTTG1 directly regulates GEF-H1 gene transcription and RhoA-GTP activation in breast cancer cells. Second, we demonstrate that activation of hPTTG1/GEF-H1 signalling is essential for breast cancer cell migration and invasion, and is required for actin cytoskeleton rearrangements upon RhoA activation. Third, we provide in vivo evidences that hPTTG1/GEF-H1 signalling is required for breast cancer metastasis in a mouse model. Finally, the 
a

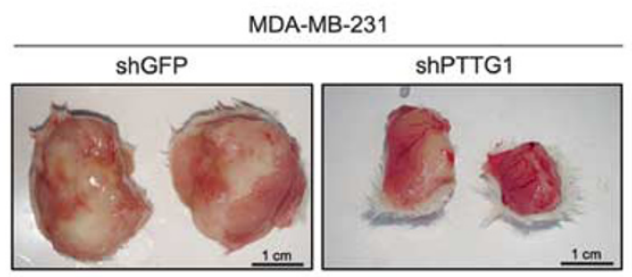

b
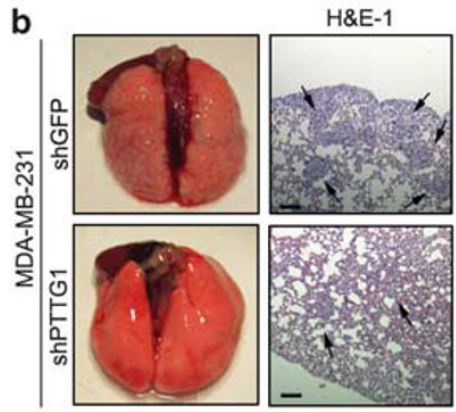

C

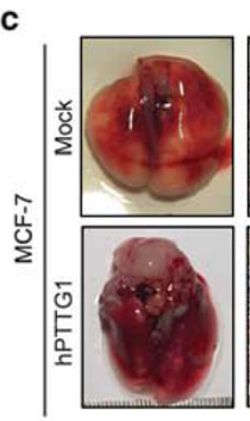

d

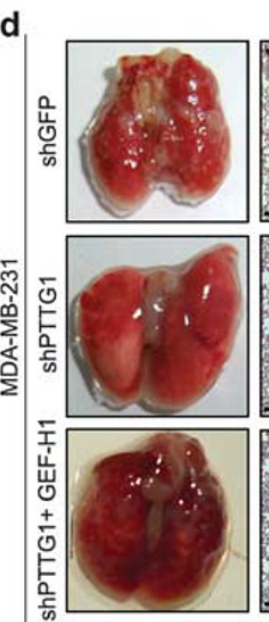

$H \& E-1$

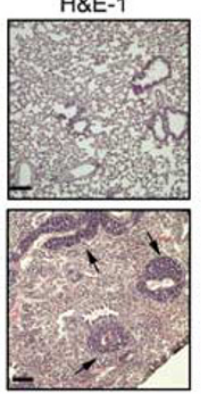

$\mathrm{H} \& \mathrm{E}-1$

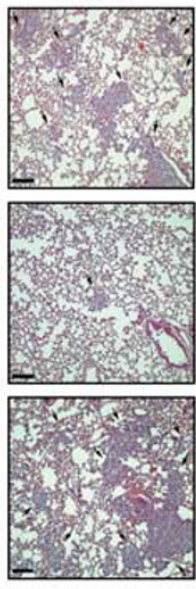

$H \& E-2$

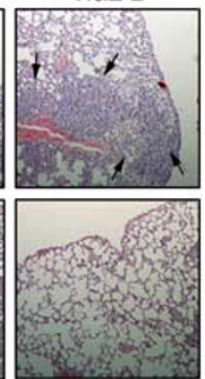

H\&E-2

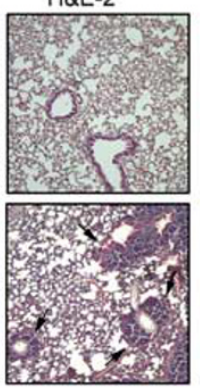

H\&E-2

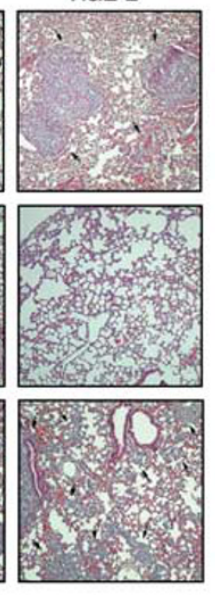

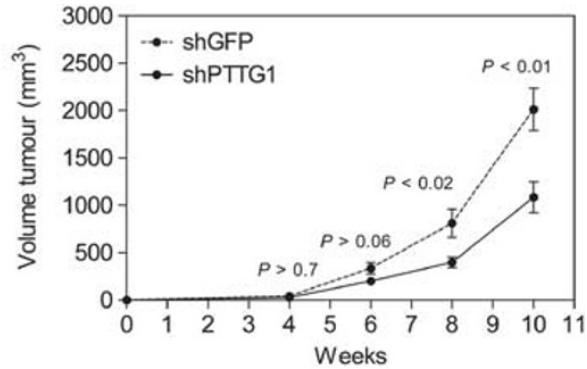
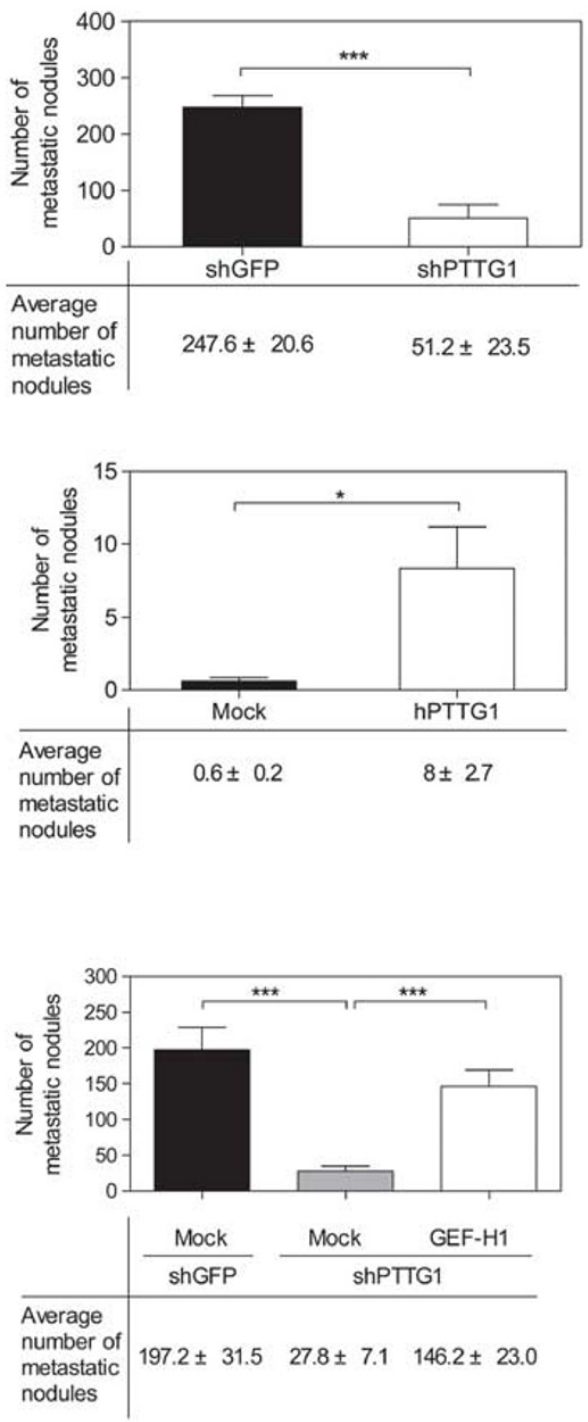

Figure 5 hPTTG1/GEF-H1 signalling regulates cancer metastasis in a mouse model. (a) Primary tumour growth was measured upon orthotopic injection of MDA-MB-231 cells with GFP or hPTTG1 knockdown (right). The experiment was terminated after 10 weeks because of primary tumour burden. Representative tumours are shown in the left panel. $n=6$ per group per time point. Each time point shows the mean \pm s.e.m. (b) Left panel: Images of murine lungs, 10 weeks after orthotopic implantation of MDA-MB-231 cells with GFP or hPTTG1 knockdown. H\&E stain of lungs from mice bearing the indicated tumours; arrows indicate metastatic foci. Right panel: Quantitative results of the lung metastasis ( $n=6$ per group). (c) Left panel: Images of murine lungs, 16 weeks after orthotopic implantation of mock-transfected or hPTTG1-overexpressed MCF-7 cells. H\&E stain of lungs from mice bearing the indicated tumours; arrows indicate metastatic foci. Right panel: Quantitative results of the lung metastasis $(n=5$ per group). (d) Left panel: Images of murine lungs 10 weeks after tail vein injection of MDA-MB-231 cells with GFP or hPTTG1 knockdown or with hPTTG1 knockdown plus GEF-H1 overexpression. H\&E stain of lungs; arrows indicate metastatic foci. Right panel: Quantitative results of the lung metastasis ( $n=6$ per group). Magnification, $\times 200$. Scale bars, $100 \mu \mathrm{m}$. The numbers of metastatic nodules in H\&E stains were counted microscopically. Data are mean \pm s.e.m. $* P<0.05 ; * * *<<0.0001$. 

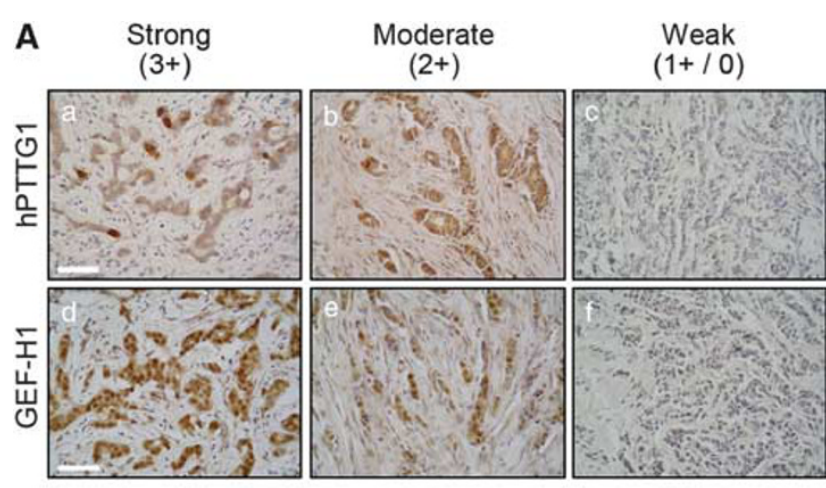

B

\begin{tabular}{lccc} 
& \multicolumn{2}{c}{ hPTTG1 } & \\
\cline { 2 - 3 } & $1+/ 0$ & $2+/ 3+$ & Total \\
\hline GEF-H1 1+/0 & 1 & 4 & 5 \\
GEF-H1 2+/3+ & 1 & 64 & 65 \\
Total & 2 & 68 & 70 \\
\hline
\end{tabular}

C

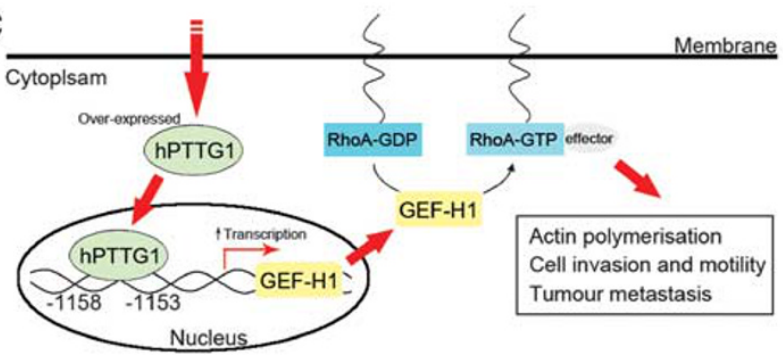

Figure 6 Positive correlation between hPTTG1 and GEF-H1 expression in invasive breast carcinoma samples. (A) The expression pattern of hPTTG1 and GEF-H1 in representative tumour tissues of patients with invasive breast carcinoma. Tissues were scored as having strong $(3+, a, d)$, moderate $(2+, b$, e), or weak expression $(1+/ 0, \mathrm{c}, \mathrm{f})$. The criteria are described in Materials and methods section. Original magnification, $\times 400$. Scale bars, $50 \mu \mathrm{m}$. (B) The expression data of hPTTG1 and GEF-H1 in 70 invasive breast carcinoma specimens (see Supplementary Figure S7b). (C) Schematic representation of the role of hPTTG1/GEF-H1/RhoA signalling in actin cytoskeletal regulation and cancer metastasis.

clinical observations show the positive correlation between hPTTG1 and GEF-H1 expression in human invasive breast cancers. This work has revealed that hPTTG1 activates GEF-H1, as a novel mechanism to regulate breast cancer metastasis via RhoA signalling.

hPTTG1, a transcription factor, can activate several genes, such as c-Myc, FGF-2, cyclin D3, p21, sodiumiodine symporter, prolactin and MMP2 (Pei, 2001; Tong and Eigler, 2009), and suggested that the transcriptional activity of hPTTG1 may have important roles in a variety of cellular processes. Several oncogenic transcription factors can promote malignant progression by induction of downstream effectors (Clevenger, 2004; Libermann and Zerbini, 2006). This concept provides a more plausible explanation as to why hPTTG1 is expressed at a high level in most malignant breast cancers. Indeed, our data show that hPTTG1 serves as a transcription factor to promote breast cancer metastasis by activating its direct target, GEF-H1. This finding is further underscored by clinical observations that tu- mour tissues of patients with invasive breast carcinoma express significant hPTTG1 nuclear staining, which is correlated to GEF-H1 expression.

There is strong evidence that both depletion and overexpression of hPTTG1 can induce apoptosis and senescence in vitro and in vivo (Chesnokova et al., 2008; Chesnokova and Melmed, 2010; Smith et al., 2010). These studies indicate that the proper amount of hPTTG1 is important for regulating physiological processes in vitro and in vivo. Senescence is considered as a first-line barrier to prevent tumour growth and malignant transformation. Genetic instabilities in cancer cells may cause mutations in important tumour suppressor genes to help cancer cells escape senescence and apoptosis. In cancer cells, impaired regulation of senescence and apoptosis may be important for hPTTG1 to exert its oncogenic properties. Overexpression of hPTTG1 can induce DNA damage response and chromosome instability (Kim et al., 2007), and overexpression seems to be an essential criterion for hPTTG1 to enhance its oncogenic potential.

RhoA-GTPase is expressed ubiquitously in different human tissues (Wennerberg and Der, 2004), and how RhoA-GTPase is activated pathologically has become an important issue in cancer biology. GEF-H1 is the major molecule that regulates the activity of RhoA physiologically. GEF-H1 activity is regulated by microtubule binding (Krendel et al., 2002), and microtubule dynamics that specifically connect cellular RhoA activation and actin cytoskeleton regulation (Birkenfeld et al., 2008). Consistent with the fact that GEF-H1 mediates the RhoA-dependent F-actin stress fibres formation (Birukova et al., 2006; Chang et al., 2008), our results show that hPTTG1-induced GEF-H1 expression increased the amount of GTP-bound RhoA, thereby increasing the assembly of F-actin stress fibres and focal adhesions in breast cancer cells. GEF-H1 has been proposed to have a role in regulating the metastatic capacities of cancer cells because its regulatory effects of actin cytoskeleton are likely to enhance the migratory and invasive properties of cancer cells (Birkenfeld et al., 2008). Such speculation is supported by our observation that the newly discovered role of hPTTG1/GEF-H1/ RhoA signalling in cytoskeleton regulation, which is one of the driving forces of breast cancer metastasis.

In this study, we provided a new insight into how hPTTG1 promotes breast cancer metastasis. Our results show that overexpressed hPTTG1 directly activates GEF-H1, which in turn, activates RhoA, a regulator of actin organisation (Figure 6C). hPTTG1 enhances the migratory and invasive ability of tumour cells through RhoA activation. Thus, hPTTG1 is an attractive therapeutic target for treating metastatic cancers.

\section{Materials and methods}

Cell lines and cell culture

MDA-MB-231, MDA-MB-435, MCF-7, BT-483, T47D, Au565, ZR-75-1 and H184B5F5/M10 cells (from ATCC, Manassas, VA, USA) were grown in Dulbecco's modified Eagle medium (DMEM) containing 10\% fetal bovine serum. HCT116 
colorectal tumour cells (from Dr Bert Vogelstein of Johns Hopkins School of Medicine) were maintained in McCoy's 5A medium containing $10 \%$ fetal bovine serum. To create pcDNA3.1-hPTTG1, hPTTG1 was PCR-amplified from the cDNA of 293T cells and then subcloned into a pcDNA3.1 vector (Invitrogen, Carlsbad, CA, USA). hPTTG1-null HCT116 and MCF-7 cells were transfected with $4 \mu \mathrm{g}$ pcDNA3.1 or pcDNA3.1hPTTG1 expression plasmid using Lipofectamine 2000 (Invitrogen) and then selected with hygromycin $(100 \mu \mathrm{g} / \mathrm{ml})$. GEF-H1 expression plasmid was obtained from ORIGENE (Rockville, MD, USA) (SC323957). MCF-7 cells were transfected with GEF$\mathrm{H} 1$ expression plasmids and then selected with neomycin $(400 \mu \mathrm{g}$ $\mathrm{ml})$. To generate control GFP-, hPTTG1- and GEF-H1-knockdown MDA-MB-231 cells, specific shRNA reagents were obtained from the National RNAi Core Facility, Academia Sinica (Taipei, Taiwan) (GFP-shRNA clone: TRCN0000072178; hPTTG1-shRNA clone: TRCN0000015103 and TRCN0000015 106). The GEF-H1-shRNA clone (TR314672) was purchased from ORIGENE. shRNA clones were selected with puromycin $(2 \mu \mathrm{g} / \mathrm{ml})$ for further use. All individual resistant colonies were isolated and characterised by immunoblot analysis.

\section{Luciferase reporter assays}

The regulatory sequence of GEF-H1 (nucleotides -1600 to +98 , which encompass 1600 bp upstream to 98 downstream of the transcription start site) was PCR-amplified from human genomic DNA, and inserted into the pGL3 luciferase vector (Promega, Madison, WI, USA). MCF-7 cells were transfected using Lipofectamine 2000, and the luciferase activities were measured $48 \mathrm{~h}$ after transfection using the Dual-Luciferase Reporter System (Promega). Site-specific mutation of the GEF-H1 promoter was performed using a QuickChange SiteDirected Mutagenesis Kit (Stratagene, Santa Clara, CA, USA) following the manufacturer's instructions.

\section{Chromatin immunoprecipitation (ChIP) assay}

The ChIP assay was carried out in accordance with the manufacturer's instructions (Millipore, Billerica, MA, USA). The chromatin preparation was sheared by sonication, and $1 \%$ of the sheared chromatin was collected as an input control. The remaining chromatin preparation was incubated with antihPTTG1 (1:100, Invitrogen) or rabbit IgG (1:500, Cell Signaling, Danvers, MA, USA) for $12 \mathrm{~h}$ at $4{ }^{\circ} \mathrm{C}$ on a rotating platform. Real-time PCR was used to enrich promoter-binding levels, and those data are expressed as fold enrichment (fold increase over the control IgG). PCR was carried out with primers as follows: GEF-H1 hPTTG1 BE-1 (binding site-1), 5'-CCCC ACCTTACCCTGCTTCTTG- $3^{\prime}$ and $5^{\prime}$-CTCCGCCTCTGCCA GCTACTTT-3'; GEF-H1 hPTTG1 BE-2, 5'-GAGCTAGGAG GAGGGCAGAGT- $3^{\prime}$ and $5^{\prime}$-GCCAGAGGGAGAGGAAAT CAA-3'; and GEF-H1 hPTTG1 BE-3, 5'-CCCGCCTTCCCCCA ACAATAC- $3^{\prime}$ and $5^{\prime}$-CACCCCTACCCACTCGCTCGCA- $3^{\prime}$.

\section{Immunoblot analysis}

The indicated cell lysates were extracted with cell lysis buffer (iNtRON), and $50 \mu \mathrm{g}$ of protein was subjected to $12 \%$ SDSpolyacrylamide gel electrophoresis analysis. Immunoblot analysis was performed with the antibodies anti-GEF-H1, RhoA (1:1000, Cell Signaling), $\beta$-actin (1:3000, Santa Cruz Biotechnology, Santa Cruz, CA, USA) and hPTTG1 (1:1000, Invitrogen).

\section{Rho $A$ activity assay}

To detect activated, GTP-bound RhoA, a RhoA-GTP purification kit was used according to the manufacturer's instructions (Millipore). Cell lysates were extracted and were used to pull down activated RhoA-GTP with a glutathione S-transferaserhotekin Rho-binding domain, which selectively bound RhoAGTP but not RhoA-GDP. After precipitation, samples were analysed by immunoblot analysis with an anti-RhoA antibody.

\section{Immunofluorescence analysis}

Cells were cultured on poly-L-lysine-coated slides for $48 \mathrm{~h}$, fixed with $4 \%$ paraformaldehyde, and permeabilised with $0.5 \%$ TritonX 100 in phosphate buffered saline (PBS). Fixed cells were blocked with $1 \%$ bovine serum albumin (BSA) in PBS and incubated with anti-paxillin (1:200, Cell Signaling) at room temperature for $1 \mathrm{~h}$. After washing three times with PBS, cells were incubated with a secondary antibody conjugated to fluorescein isothiocyanate (FITC) and Alexa flour 555-conjugated phalloidin (Invitrogen) for $1 \mathrm{~h}$ at room temperature. Nuclei were visualised with $4^{\prime}, 6^{\prime}$ diamino-2-phenylindole (DAPI). Morphological alterations, actin filament formation and paxillin puncta in the cells were observed, and images were captured with a confocal microscope (LEICA TCS SP2 AOBS, LEICA, Wetzlar, Germany).

\section{Migration and invasion assays}

For the migration assays, $2.5 \times 10^{4}$ cells were seeded in an uncoated membranes, with $8.0 \mu \mathrm{m}$ pores (Corning, Corning, NY, USA); for invasion assays, $1 \times 10^{5}$ cells were seeded in a Matrigelcoated chamber, with $8.0 \mu \mathrm{m}$ pores (BD Bioscience, Franklin Lakes, NJ, USA). Cells were seeded in a serum-free medium and migrated toward complete growth media for $12-15 \mathrm{~h}$. The migrating or invading cells in polycarbonate membranes were stained and counted as previous reported (Chan et al., 2010).

\section{Animal studies}

For spontaneous metastasis assay, $2 \times 10^{5}$ tumour cells were injected into the fourth mammary fat pad of 5- to 8-week-old SCID mice in 1:1 Matrigel (BD Bioscience) plus PBS. Primary tumour growth upon orthotopic injection was obtained by weekly measurements. For the experimental metastasis assay, $1 \times 10^{6}$ tumour cells were injected into the tail vein of 5- to 8-week-old SCID mice. All dissected lungs were paraffin-embedded, sectioned, stained with haematoxylin and eosin (H\&E), and metastatic nodules counted by microscope (4-5 lobes per lung in a mouse). An average of metastatic nodules per lung in mice was shown in Figures $5 \mathrm{~b}-\mathrm{d}$ (right panels). All animals were maintained in the Laboratory Animal Center at Tzu-Chi University.

\section{Immunohistochemistry (IHC)}

Human breast cancer tissue samples (70 patients) were collected with approval from Tzu-Chi Hospital for immunohistochemistry analysis. Paraffin-embedded tissue sections $(3 \mu \mathrm{m})$ were immunostained following a previously described protocol (Liu et al., 2008), with the antibodies anti-hPTTG1 (1:100, Invitrogen), anti-GEFH1 (1:40, ATLAS Antibody, Stockholm, Sweden) and RhoA (1:100, Proteintech, Chicago, IL, USA). Nuclei were counterstained with haematoxylin. To evaluate the expression levels of hPTTG1 and GEF-H1, immunostained human breast tissues were judged by two pathologists using the following criteria. hPTTG1 was classified as $3+$ (strong hPTTG1 nuclear staining in $>5 \%$ tumour cells or moderate hPTTG1 nuclear staining in $>50 \%$ tumour cells), $2+$ (moderately positive hPTTG1 nuclear staining in $<50 \%$ tumour cells), and $0 / 1+$ (no or weak hPTTG1 nuclear staining), and GEF-H1 was classified as strong $(3+)$, moderate $(2+)$ or weak $(0 / 1+)$ based on the intensity of both nuclear and cytoplasmic immunoreactivity. The association of hPTTG1 expression with GEF-H1 expression in invasive breast carcinoma was analyzed by Pearson's correlation test. 
Statistical analysis

Data are presented as mean \pm s.e.m. unless otherwise noted. Student's $t$-test was used for comparisons. $P<0.05$ was considered significant.

\section{Conflict of interest}

The authors declare no conflict of interest.

\section{Acknowledgements}

Ms Lily Chen, Ms Vicky Chang and Ms Shi-Rong Lin provided expert technical assistance. We thank Dr Lu-Hai Wang's suggestions during the preparation of this manuscript. This study was supported by grants from the National Science Council of Taiwan (NSC96-2320-B-320-013-MY3), the National Health Research Institutes of Taiwan (NHRIEX99-9928BI) and also partially by the Tzu-Chi Foundation.

\section{References}

Bernal J, Luna R, Espina A, La?zaro I, Ramos-Morales F, Romero F et al. (2002). Human securin interacts with p53 and modulates p53-mediated transcriptional activity and apoptosis. Nat Genet 32: 306-311.

Birkenfeld J, Nalbant P, Yoon S, Bokoch G. (2008). Cellular functions of GEF-H1, a microtubule-regulated Rho-GEF: is altered GEF-H1 activity a crucial determinant of disease pathogenesis? Trends Cell Biol 18: 210-219.

Birukova AA, Adyshev D, Gorshkov B, Bokoch GM, Birukov KG, Verin AD. (2006). GEF-H1 is involved in agonist-induced human pulmonary endothelial barrier dysfunction. Am J Physiol Lung Cell Mol Physiol 290: L540-L548.

Chambers AF, Groom AC, MacDonald IC. (2002). Dissemination and growth of cancer cells in metastatic sites. Nat Rev Cancer 2 : 563-572.

Chan $\mathrm{CH}$, Lee SW, Li CF, Wang J, Yang WL, Wu CY et al. (2010). Deciphering the transcriptional complex critical for RhoA gene expression and cancer metastasis. Nat Cell Biol 12: 457-467.

Chang Y, Nalbant P, Birkenfeld J, Chang Z, Bokoch G. (2008). GEF-H1 couples nocodazole-induced microtubule disassembly to cell contractility via RhoA. Mol Biol Cell 19: 2147-2153.

Chesnokova V, Melmed S. (2010). Pituitary senescence: the evolving role of Pttg. Mol Cell Endocrinol 326: 55-59.

Chesnokova V, Zonis S, Kovacs K, Ben-Shlomo A, Wawrowsky K, Bannykh S et al. (2008). p21(Cip1) restrains pituitary tumor growth. Proc Natl Acad Sci USA 105: 17498-17503.

Chien W, Pei L. (2000). A novel binding factor facilitates nuclear translocation and transcriptional activation function of the pituitary tumor-transforming gene product. J Biol Chem 275 : 19422-19427.

Clevenger CV. (2004). Roles and regulation of stat family transcription factors in human breast cancer. Am J Pathol 165: 1449-1460.

del Peso L, Hernandez-Alcoceba R, Embade N, Carnero A, Esteve P, Paje C et al. (1997). Rho proteins induce metastatic properties in vivo. Oncogene 15: 3047-3057.

Dominguez A, Ramos-Morales F, Romero F, Rios RM, Dreyfus F, Tortolero M et al. (1998). hpttg, a human homologue of rat pttg, is overexpressed in hematopoietic neoplasms. Evidence for a transcriptional activation function of hPTTG. Oncogene 17: 2187-2193.

Fidler IJ. (2003). The pathogenesis of cancer metastasis: the 'seed and soil' hypothesis revisited. Nat Rev Cancer 3: 453-458.

Funabiki H, Kumada K, Yanagida M. (1996). Fission yeast Cut1 and Cut 2 are essential for sister chromatid separation, concentrate along the metaphase spindle and form large complexes. EMBO $J \mathbf{1 5}$ : $6617-6628$.

Gil-Bernabe AM, Romero F, Limon-Mortes MC, Tortolero M. (2006). Protein phosphatase 2A stabilizes human securin, whose phosphorylated forms are degraded via the SCF ubiquitin ligase. Mol Cell Biol 26: 4017-4027.

Gupta GP, Massague J. (2006). Cancer metastasis: building a framework. Cell 127: 679-695.

Hall A. (1998). Rho GTPases and the actin cytoskeleton. Science 279: $509-514$.

Heaney A, Singson R, McCabe C, Nelson V, Nakashima M, Melmed S. (2000). Expression of pituitary-tumour transforming gene in colorectal tumours. Lancet 355: 716-719.

Hornig NC, Knowles PP, McDonald NQ, Uhlmann F. (2002). The dual mechanism of separase regulation by securin. Curr Biol 12: 973-982.

Ito T, Shimada Y, Kan T, David S, Cheng Y, Mori Y et al. (2008). Pituitary tumor-transforming 1 increases cell motility and promotes lymph node metastasis in esophageal squamous cell carcinoma. Cancer Res 68: 3214-3224.

Jaffe AB, Hall A. (2005). Rho GTPases: biochemistry and biology. Annu Rev Cell Dev Biol 21: 247-269.

Kim DS, Franklyn JA, Smith VE, Stratford AL, Pemberton HN, Warfield A et al. (2007). Securin induces genetic instability in colorectal cancer by inhibiting double-stranded DNA repair activity. Carcinogenesis 28: 749-759.

Krendel M, Zenke F, Bokoch G. (2002). Nucleotide exchange factor GEF-H1 mediates cross-talk between microtubules and the actin cytoskeleton. Nat Cell Biol 4: 294-301.

Libermann TA, Zerbini LF. (2006). Targeting transcription factors for cancer gene therapy. Curr Gene Ther 6: 17-33.

Liu YN, Liu Y, Lee HJ, Hsu YH, Chen JH. (2008). Activated androgen receptor downregulates E-cadherin gene expression and promotes tumor metastasis. Mol Cell Biol 28: 7096-7108.

Mizuarai S, Yamanaka K, Kotani H. (2006). Mutant p53 induces the GEF-H1 oncogene, a guanine nucleotide exchange factor-H1 for RhoA, resulting in accelerated cell proliferation in tumor cells. Cancer Res 66: 6319-6326.

Ogbagabriel S, Fernando M, Waldman FM, Bose S, Heaney AP. (2005). Securin is overexpressed in breast cancer. Mod Pathol 18: 985-990.

Pei L. (2001). Identification of c-myc as a down-stream target for pituitary tumor-transforming gene. $J$ Biol Chem 276: 8484-8491.

Pei L, Melmed S. (1997). Isolation and characterization of a pituitary tumor-transforming gene (PTTG). Mol Endocrinol 11: 433-441.

Ramaswamy S, Ross K, Lander E, Golub T. (2003). A molecular signature of metastasis in primary solid tumors. Nat Genet 33: 49-54.

Ren Y, Li R, Zheng Y, Busch H. (1998). Cloning and characterization of GEF-H1, a microtubule-associated guanine nucleotide exchange factor for Rac and Rho GTPases. J Biol Chem 273: 34954-34960.

Ridley AJ. (2001). Rho GTPases and cell migration. J Cell Sci 114: 2713-2722.

Romero F, Multon MC, Ramos-Morales F, Dominguez A, Bernal JA, Pintor-Toro JA et al. (2001). Human securin, hPTTG, is associated with $\mathrm{Ku}$ heterodimer, the regulatory subunit of the DNA-dependent protein kinase. Nucleic Acids Res 29: 1300-1307.

Sahai E, Marshall CJ. (2002). RHO-GTPases and cancer. Nat Rev Cancer 2: 133-142.

Schaller MD. (2001). Paxillin: a focal adhesion-associated adaptor protein. Oncogene 20: 6459-6472.

Schmitz AA, Govek EE, Bottner B, Van Aelst L. (2000). Rho GTPases: signaling, migration, and invasion. Exp Cell Res 261: 1-12. 
Smith VE, Franklyn JA, McCabe CJ. (2010). Pituitary tumortransforming gene and its binding factor in endocrine cancer. Expert Rev Mol Med 12: e38.

Solbach C, Roller M, Fellbaum C, Nicoletti M, Kaufmann M. (2004). PTTG mRNA expression in primary breast cancer: a prognostic marker for lymph node invasion and tumor recurrence. Breast 13: $80-81$.

Tong Y, Eigler T. (2009). Transcriptional targets for pituitary tumortransforming gene-1. J Mol Endocrinol 43: 179-185.

Valastyan S, Reinhardt F, Benaich N, Calogrias D, Szasz AM, Wang ZC et al. (2009). A pleiotropically acting microRNA, miR-31, inhibits breast cancer metastasis. Cell 137: 1032-1046.

Vlotides G, Eigler T, Melmed S. (2007). Pituitary tumor-transforming gene: physiology and implications for tumorigenesis. Endocr Rev 28: 165-186.

Wang Z, Melmed S. (2000). Pituitary tumor transforming gene (PTTG) transforming and transactivation activity. $\mathrm{J}$ Biol Chem 275: 7459-7461.

Wennerberg K, Der CJ. (2004). Rho-family GTPases: it's not only Rac and Rho (and I like it). J Cell Sci 117: 1301-1312.

Wittmann T, Waterman-Storer CM. (2001). Cell motility: can Rho GTPases and microtubules point the way? J Cell Sci 114: 37953803.

Zhang X, Horwitz GA, Prezant TR, Valentini A, Nakashima M, Bronstein MD et al. (1999). Structure, expression, and function of human pituitary tumor-transforming gene (PTTG). Mol Endocrinol 13: 156-166.

Zou H, McGarry TJ, Bernal T, Kirschner MW. (1999). Identification of a vertebrate sister-chromatid separation inhibitor involved in transformation and tumorigenesis. Science 285: 418-422.

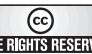

SOMERIEHTS RESER

sor visit http://creativecommons.org/licenses/by-nc-nd/3.0/

Supplementary Information accompanies the paper on the Oncogene website (http://www.nature.com/onc) 